\title{
Evening not morning plasma cortisol level is higher in women with polycystic ovary syndrome.
}

Authors: Marta Kiałka MD¹, Tomasz Milewicz PhD¹, Agnieszka Ociepka MD²; Magdalena Krzyczkowska- Sendrakowska MD' ,Ewa Stochmal MD²

Hospital: ${ }^{1}$ Department of Gynecological Endocrinology, Jagiellonian University, Medical College, Cracow, Poland 2Department of Endocrinology, Jagiellonian University, Medical College, Cracow, Poland

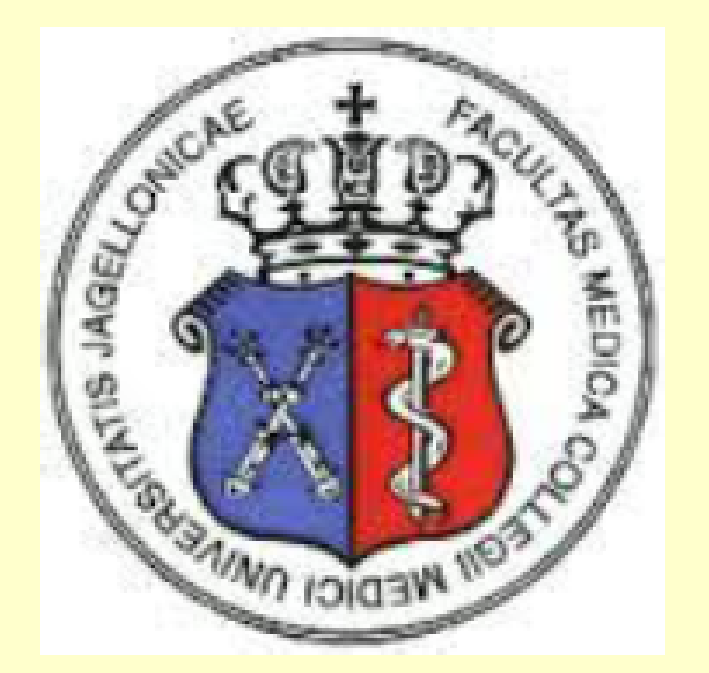

\section{OBJECTIVES}

The aim of our study was to assess the morning and evening cortisol plasma levels in women with polycystic ovary syndrome (PCOS).
Table 1. Clinical characteristics of PCOS patients (group A) and controls (group B).

\begin{tabular}{|l|l|l|l|}
\hline Parameter & $\begin{array}{l}\text { Group A } \\
\mathrm{n}=40\end{array}$ & $\begin{array}{l}\text { Group B } \\
\mathrm{n}=55\end{array}$ & $\mathrm{P}$ \\
\hline Age (years) & $27.3 \pm 3.6$ & $29.8 \pm 6.8$ & $\mathrm{NS}$ \\
Range & $(20-32)$ & $(15-36)$ & \\
\hline BMI $\left(\mathrm{kg} / \mathrm{m}^{2}\right)$ & $24.1 \pm 6.5$ & $25.7 \pm 7.2$ & NS \\
Range & $(17.8-42.3)$ & $(16.5-44.8)$ & \\
\hline
\end{tabular}

Table 2. Hormonal characteristics of PCOS patients (group A) and controls (group B).

\begin{tabular}{|c|c|c|c|}
\hline parameter & $\begin{array}{l}\text { Group A } \\
n=40\end{array}$ & $\begin{array}{l}\text { Group B } \\
n=55\end{array}$ & $\mathrm{P}$ \\
\hline $\begin{array}{l}\text { LH (IU/l) } \\
\text { Range }\end{array}$ & $\begin{array}{l}10.7 \pm 6.8 \\
(4.5-29.0)\end{array}$ & $\begin{array}{l}6.6 \pm 4.5 \\
(2.2-19)\end{array}$ & $<0.02$ \\
\hline $\begin{array}{l}\text { FSH (IU/l) } \\
\text { Range }\end{array}$ & $\begin{array}{l}6.2 \pm 1.7 \\
(1.6-8.8)\end{array}$ & $\begin{array}{l}5.7 \pm 2.0 \\
(2-9.9)\end{array}$ & NS \\
\hline $\begin{array}{l}\text { Estradiol (ng/l) } \\
\text { Range }\end{array}$ & $\begin{array}{l}76.5 \pm 14.0 \\
(37.9-99.0)\end{array}$ & $\begin{array}{l}86.9 \pm 103.7 \\
30.8=537.0 \\
)\end{array}$ & NS \\
\hline $\begin{array}{l}\text { Prolactin (ug/l) } \\
\text { Range }\end{array}$ & $\begin{array}{l}13.3 \pm 6.4 \\
(4.9-28.1)\end{array}$ & $\begin{array}{l}14.2 \pm 11.3 \\
(2.4-28.0)\end{array}$ & NS \\
\hline $\begin{array}{l}\text { Testosterone } \\
(\mathrm{nmol} / \mathrm{l}) \\
\text { Range }\end{array}$ & $\begin{array}{l}3.8 \pm 0.6 \\
(3.1-5.5)\end{array}$ & $\begin{array}{l}1,6 \pm 0,6 \\
(0.3-2.7)\end{array}$ & $<0.001$ \\
\hline $\begin{array}{l}\text { DHEAS } \\
(\mathrm{nmol} / \mathrm{l}) \\
\text { range }\end{array}$ & $\begin{array}{l}427.7 \pm 162.9 \\
(148.1-647.8)\end{array}$ & $\begin{array}{l}236.6 \pm 97.8 \\
(95-436.0)\end{array}$ & $<0.001$ \\
\hline $\begin{array}{l}\text { SHBG }(\mathrm{ng} / \mathrm{ml}) \\
\text { Range }\end{array}$ & $\begin{array}{l}65.4 \pm 36.6 \\
(14.6-138.3)\end{array}$ & $\begin{array}{l}63.8 \pm 36.4 \\
(14.5- \\
132.8) \\
\end{array}$ & NS \\
\hline $\begin{array}{l}\text { TSH (uIU/ml) } \\
\text { Range }\end{array}$ & $\begin{array}{l}1.6 \pm 0.7 \\
(0.5-3.0)\end{array}$ & $\begin{array}{l}2.1 \pm 1.2 \\
(1.0-3.9)\end{array}$ & NS \\
\hline
\end{tabular}

\section{METHODS}

95 patients gave their informed consent to participate in the study and were divided into 2 groups. Group A consisted of 40 PCOS patients and group B consisted of 55 women without features of PCOS. Between day 5 and 8 of the menstrual cycle, morning $(7 \mathrm{am})$, fasting blood samples were taken for the assessment of luteinizng hormone (LH), follicle stimulating hormone (FSH), estradiol, cortisol, prolactin, thyreotropin (TSH), testosterone and dehydroepeiandrosterone (DHEAS). Evening $(5 \mathrm{pm})$ blood samples were also taken for the evaluation of plasma cortisol level

\section{RESULTS}

There were no differences in mean age, body mass index (BMI), FSH, SHBG, PRL, estradiol and TSH levels between group A and group B. Mean plasma LH level was higher in group A compared to group $B(10.7 \pm 6.8 \mathrm{IU} / \mathrm{l}$ vs $6.6 \pm 4.5 \mathrm{IU} / \mathrm{I}, \mathrm{p}<0.02)$. Mean plasma testosterone and DHEAS levels were also higher in PCOS patients $(3.8 \pm 0.6 \mathrm{nmol} / \mathrm{l}$ vs $1.63 \pm 0.6 \mathrm{nmol} / \mathrm{l} ; 427.7 \pm 162.9$ vs $236.6 \pm 97.8$ resp , $p<0.001))$. Mean evening plasma cortisol level was higher in PCOS patients $(11.8 \pm 4.1 \mathrm{ug} / \mathrm{dl}$ vs $7.4 \pm 5.0 \mathrm{ug} / \mathrm{dl}, \mathrm{p}<0.02)$. Mean morning plasma cortisol levels did not differ between groups.

\section{CONCLUSIONS}

PCOS women showed the increased plasma cortisol level with impacted diurnal secretion rate.
Table 3. Plasma cortisol levels in PCOS patients (group A) and in controls (group B).

\begin{tabular}{|l|l|l|l|}
\hline & $\begin{array}{l}\text { Group A } \\
\mathrm{n}=40\end{array}$ & $\begin{array}{l}\text { Group B } \\
\mathrm{n}=55\end{array}$ & $\mathrm{P}$ \\
\hline $\begin{array}{l}\text { Cortisol 7 am } \\
\text { (ug/dl) } \\
\text { range }\end{array}$ & $\begin{array}{l}14.6 \pm 9.8 \\
(2.5-28.1)\end{array}$ & $\begin{array}{l}14.2 \pm 10.0 \\
(2.0-25.3)\end{array}$ & $\mathrm{NS}$ \\
\hline $\begin{array}{l}\text { Cortisol } 5 \mathrm{pm} \\
\text { (ug/dl) } \\
\text { Range }\end{array}$ & $\begin{array}{l}11.8 \pm 1,1 \\
(2.6-7.7)\end{array}$ & $\begin{array}{l}4.7 \pm 1.3 \\
(2.9-7.7)\end{array}$ & $<0.02$ \\
\hline
\end{tabular}

data is presented as mean \pm standard deviation (SD) and minimal and maximal value.

\section{References}

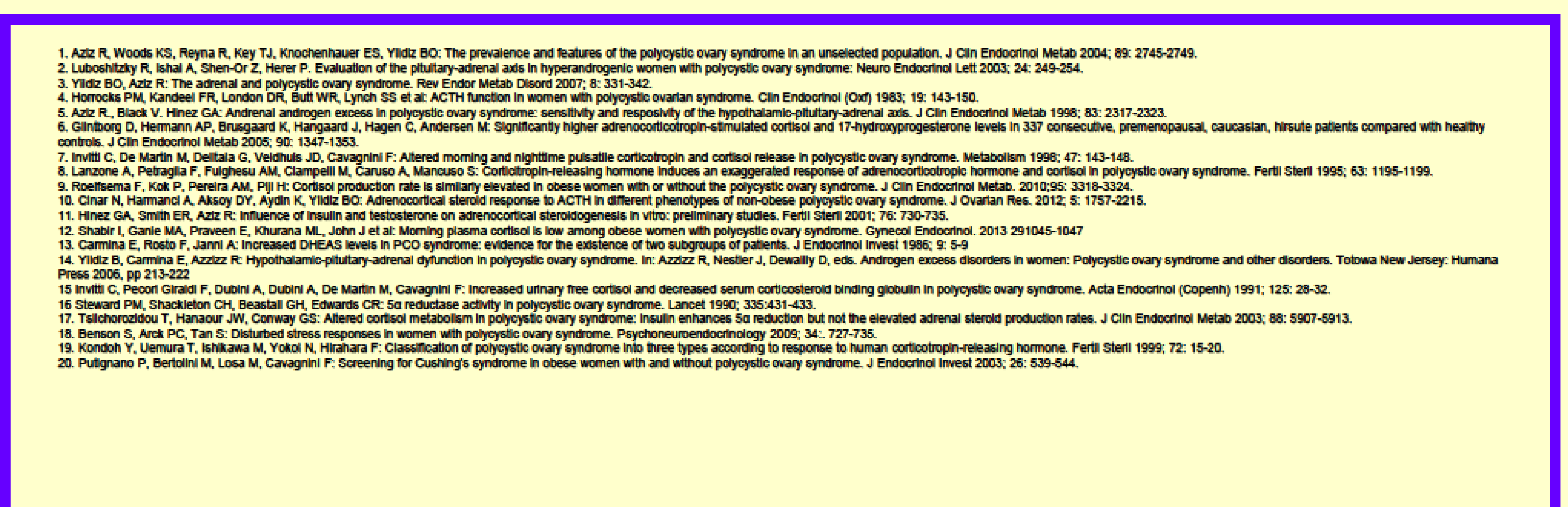

